Alexandra Popa

Holistic Dental \& Medical Institute of Bucharest-ROPOSTURO, Bucharest, Romania

e-mail:

alesandra17popa@gmail.com

\section{Dental Digital Photography From Dental Clinical Photography to Digital Smile Design}

Editors: Feng Liu

Publisher: Springer Nature Singapore

Language: English

ISBN: 978-981-13-1622-7

Edition: $1 / \mathrm{e}$

Publish Year: 2019

Pages: 318, illustrated

Price: $160,49 €$

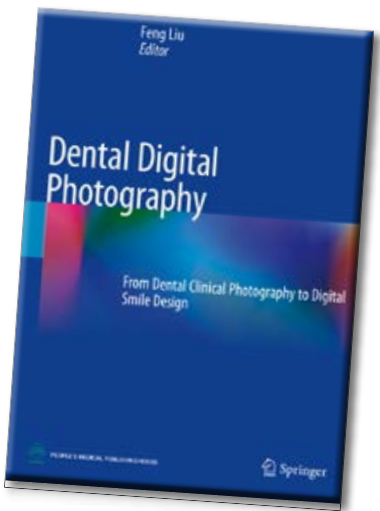

Medical photography in clinical practice has been widely used in oral medicine in recent years. Using clinical photography, a dentist can record a patient's case with greater medical accuracy and schedule appropriate treatment.

This revised third edition of Dental Digital Photography covers 9 topics.

The book begins with the presentation of The History and Significance of Dental Photography and explains the importance of photos in patient education, preserving the images for medical records, the doctor-patient, doctor-technician and doctor-doctor communications, doctor-technician and doctor-doctor as well as the legal basis. The next part tackles the Basic Concepts and Knowledge of the Photography. Prior to learning oral clinical photography, it is necessary for learners to understand the basic concepts and knowledge of photography. So, there are exhaustive explanations of notions such as photoelectric sensor, pixel and resolution, exposure, aperture, shutter speed, exposure control, ISO, depth of field, layout and white balance. The next topic addressed is Devices and Equipment of the Dental Photography. This part helps us to choose the appropriate equipment and devices to achieve ideal dental photography. Then the Basic Procedures of Dental Clinical Photography are presented. This part describes the dental photography protocol such as clinical image specification recommended, position and holding, placing retractors, composition and focus.

Basic Images of Dental Clinical Photography Considering is the next chapter. It summarizes 74 images usually used in clinical work. The shooting methods and applications of all these images are introduced in detail in this chapter, which describing both photographs taken with the camera and photos taken with the microscope. The next chapter, Standards for Common Aesthetic Oral Clinical Photography introduces the three main standards required by the: American Academy of Cosmetic Dentistry (AACD), European Society of Cosmetic Dentistry (ESCD) and Chinese Society of Aesthetic Dentistry (CSED). Clinical Still Life and Operation Photography is the following chapter. Besides clinical photographs there are also many other photos necessary in a clinic, such as photographs of clinical objects and photographs of clinical operations. Some examples are photographs of impressions, models and prostheses. Image Adjustment and Application is the next part. The chapter focuses on how to adjust images by some simple application of Photoshop, and the procedure of introducing acquired clinical images into the Digital Smile Design (DSD) process. The last part, Aesthetic Treatment Based on Clinical Image presents the basic protocol to establish aesthetic goals and it is explained by presenting many clinical cases.

The third edition of Dental Digital Photography is more comprehensiveand systematic providing a greater sense of knowledge. It will contribute to a clearer understanding of the scientific oral clinical photography concepts and methods, thus becoming for each dentist a working tool in the daily application of digital photography.

DOI: http://www.stomaeduj.com 10.25241/stomaeduj.2020.7(1).bookreview.4

The Books Review is drafted in the reviewer's sole wording and illustrates his opinions. 